\title{
THE WEAK-STAR CLOSURE OF THE UNIT BALL IN A HYPERPLANE
}

\author{
by G. J. O. JAMESON \\ (Received 25th March 1971)
}

\section{Introduction}

Let $X$ be a normed linear space. We regard $X$ as a subspace of its bidual $X^{* *}$. Polars will always be evaluated in the pair $\left(X^{* *}, X^{*}\right)$. We denote the closed unit ball in $X$ by $U$, so that $U^{0}, U^{00}$ are the closed unit balls in $X^{*}$, $X^{* *}$ respectively. The weak topology induced by $X$ on $X^{*}$ (the "weak-star" topology) will be denoted by $\sigma(X)$, and $\mathrm{cl}()$ will denote $\sigma(X)$-closure.

Let $\phi$ be an element of $X^{* *}$ that is not in $X$, and let $K$ be the kernel of $\phi$. Then $K$ is, of course, $\sigma(X)$-dense in $X^{*}$. When $X$ is complete, the KreinSmul'yan theorem tells us that $K \cap U^{0}$ is not $\sigma(X)$-closed, but it gives no further information about the set $\operatorname{cl}\left(K \cap U^{0}\right)$. The purpose of this note is to determine $\operatorname{cl}\left(K \cap U^{0}\right)$ as accurately as possible (in doing so, we shall obtain incidentally a very simple proof of the Krein-Smul'yan theorem for hyperplanes). The radius of the largest ball contained in $\operatorname{cl}\left(K \cap U^{0}\right)$ is known to be

$$
\inf \{\|x-\lambda \phi\|: x \in X,\|x\|=1, \lambda \text { scalar }\}
$$

((1), ch. IV, $\S 5$, ex. 14). This last statement applies, in fact, when $K$ is any $\sigma(X)$-dense linear subspace of $X^{*}$ (with elements of $K^{0}$ replacing the multiples $\lambda \phi)$, and is generalised to arbitrary linear subspaces in (3). However, $\operatorname{cl}\left(K \cap U^{0}\right)$ is clearly not just a multiple of $U^{0}$. Denoting by $d(\phi, X)$ the norm-distance from $\phi$ to $X$, we shall prove that

where

$$
A(\phi) \subseteq \operatorname{cl}\left(K \cap U^{0}\right) \subseteq B(\phi),
$$

$$
\begin{aligned}
& A(\phi)=\left\{f \in X^{*}:\|f\|+\frac{|\langle\phi, f\rangle|}{d(\phi, X)} \leqq 1\right\}, \\
& B(\phi)=\left\{f \in U^{0}:|\langle\phi, f\rangle| \leqq 2 d(\phi, X)\right\} .
\end{aligned}
$$

In the particular case $X=c_{0}$, we show that $\operatorname{cl}\left(K \cap U^{0}\right)$ is always $A(\phi)$. In general, however, $\operatorname{cl}\left(K \cap U^{0}\right)$ can be either $A(\phi), B(\phi)$ or something between the two.

The appearance of the ratio $\langle\phi, f\rangle \mid d(\phi, X)$ in the description is not as unreasonable as may at first seem, as the following considerations suggest:

(1) One would expect $f$ to have a better chance of being in $\operatorname{cl}\left(K \cap U^{0}\right)$ if $\langle\phi, f\rangle \mid$ is small. 
(2) $K$ is unchanged if $\phi$ is multiplied by a non-zero scalar. Hence the factor $|\langle\phi, f\rangle|$ will need to be balanced by something else that is multiplied by $|\lambda|$ when $\phi$ is replaced by $\lambda \phi$.

(3) If $d(\phi, X)$ is small, then $\phi$ is not far from being $\sigma(X)$-continuous. Consequently, one might expect $\operatorname{cl}\left(K \cap U^{0}\right)$ to be small.

The author is indebted to the referee for suggesting a better proof of Theorem 1, and for the comment in Note 3 to Theorem 1.

\section{The theorems}

Theorem 1. If $d(\phi, X)>0$, then $\operatorname{cl}\left(K \cap U^{\circ}\right)$ contains $A(\phi)$.

Proof. For the moment, let $K$ be any linear subspace of $X^{*}$. It follows at once from the Hahn-Banach theorem, by extending the restriction to $K$, that

$$
\left(K \cap U^{0}\right)^{0}=K^{0}+U^{00} \text {. }
$$

Hence $\operatorname{cl}\left(K \cap U^{0}\right)$ is the polar (in $\left.X^{*}\right)$ of $X \cap\left(K^{0}+U^{00}\right)$. If $K$ is now the kernel of $\phi$, then $K^{0}$ is the linear span of $\phi$. The result follows if we show that, for any $f$ in $X^{*}$,

$$
\sup \left\{|\langle x, f\rangle|: x \in X \cap\left(K^{0}+U^{\circ 0}\right)\right\} \leqq\|f\|+\frac{|\langle\phi, f\rangle|}{d(\phi, X)} .
$$

Let $x$ be in $X \cap\left(K^{0}+U^{00}\right)$. Then there exist $\psi$ in $U^{00}$ and a scalar $\lambda$ such that $x=\lambda \phi+\psi$. Then $\|\lambda \phi-x\| \leqq 1$, so $|\lambda| \leqq 1 / d(\phi, X)$, and

$$
|\langle x, f\rangle|=|\langle\lambda \phi+\psi, f\rangle| \leqq|\lambda| \cdot|\langle\phi, f\rangle|+\|f\|,
$$

giving the required inequality.

\section{Notes}

(1) In particular, if $d(\phi, X)>0$, then $K \cap U^{0}$ is not $\sigma(X)$-closed. Hence we have proved the Krein-Šmul'yan theorem for hyperplanes: if $X$ is complete and $K \cap U^{0}$ is $\sigma(X)$-closed, then $K$ is $\sigma(X)$-closed.

(2) Similar reasoning can be applied to the common kernel of a finite number of functionals. Let $\phi_{1}, \ldots, \phi_{m}$ be elements of $X^{* *}$ such that

$$
\inf \left\{\left\|\sum_{i=1}^{m} \lambda_{i} \phi_{i}-x\right\|: x \in X, \sum_{i=1}^{m}\left|\lambda_{i}\right|=1\right\}=r>0,
$$

and let $K=\bigcap_{i=1}^{m} \operatorname{ker} \phi_{i}$. Then $\operatorname{cl}\left(K \cap U^{0}\right)$ contains

$$
\left\{f \in X^{*}:\|f\|+\frac{1}{r} \max \left|\left\langle\phi_{i}, f\right\rangle\right| \leqq 1\right\} \text {. }
$$

For if $L$ is the linear span of $\phi_{1}, \ldots, \phi_{m}$, and $x$ is in $X \cap\left(L+U^{00}\right)$, then it is easily seen that

$$
|\langle x, f\rangle| \leqq\|f\|+\frac{1}{r} \max \left|\left\langle\phi_{i}, f\right\rangle\right|
$$


(3) Let $K$ be a linear subspace of $X^{*}$. Using the weak compactness of $U^{00}$, it is easy to show that

$$
X \cap\left(K^{0}+U^{00}\right)=\left\{x \in X: d\left(x, K^{0}\right) \leqq 1\right\} .
$$

Hence $\operatorname{cl}\left(K \cap U^{0}\right)$ is precisely the dual unit ball when $X$ is given the seminorm $p$, where $p(x)=d\left(x, K^{0}\right)$. This, of course, is the seminorm induced on $X$ by the quotient norm in $X / K^{0}$. It is a norm when $K$ is $\sigma(X)$-dense in $X^{*}$.

(4) The author's original proof of Theorem 1 was similar to the proof of the related result Corollary II, 4, 3 in (2). The proof given above was suggested by the referee.

Theorem 2. $\operatorname{cl}\left(K \cap U^{0}\right)$ is contained in $B(\phi)$.

Proof. $\mathrm{cl}\left(K \cap U^{0}\right)$ is contained in $U^{0}$, since $U^{0}$ is $\sigma(X)$-closed. Write $d(\phi, X)=r$. Suppose that $f \in U^{0}$ and $|\langle\phi, f\rangle|>2 r$ : let $|\langle\phi, f\rangle|=2 r+3 \alpha$. Take $x_{0} \in X$ such that $\left\|\phi-x_{0}\right\|<r+\alpha$. Then $\left|\left\langle\phi-x_{0}, f\right\rangle\right|<r+\alpha$, since $\|f\| \leqq 1$, so $\left|\left\langle x_{0}, f\right\rangle\right|>r+2 \alpha$. For $g$ in $U^{0},\left|\left\langle\phi-x_{0}, g\right\rangle\right|\langle r+\alpha$, so if

$$
\left|\left\langle x_{0}, g\right\rangle\right|>r+\alpha,
$$

then $g \notin K$. Hence $\left\{g \in X^{*}:\left|\left\langle x_{0}, g-f\right\rangle\right| \leqq \alpha\right\}$ is disjoint from $K \cap U^{0}$.

Corollary. If $d(\phi, X)=0$, then $K \cap U^{0}$ is $\sigma(X)$-closed.

Hence if $X$ is an incomplete normed space, and $\phi$ is an element of $X^{* *} \backslash X$ with $d(\phi, X)=0$, then $K \cap U^{0}$ is $\sigma(X)$-closed, though $K$ is $\sigma(X)$-dense in $X^{*}$ (a fact noted by Kerr (4)).

The set $B(\phi)$ is not necessarily $\sigma(X)$-closed (cf. examples below).

\section{Two particular cases}

(i) $X=c_{0}$. We show that, in this case, $\operatorname{cl}\left(K \cap U^{0}\right)$ is always equal to $A(\phi)$. Identify $c_{0}^{*}$ with $l_{1}$ and $c_{0}^{* *}$ with $m$. We use the notation $x(n)$ for the $n$th term of a sequence $x$; we continue to use the notation $\langle$,$\rangle for the evaluation$ of functionals. It is sufficient to consider $\phi \in m$ with $d\left(\phi, c_{0}\right)=1$. Take an element $f$ of $l_{1}$ that is not in $A(\phi)$ : then

$$
\|f\|+|\langle\phi, f\rangle|=1+3 \alpha
$$

for some $\alpha>0$. Since $d\left(\phi, c_{0}\right)=1$, there exists $N$ such that $|\phi(i)| \leqq 1+\alpha$ for all $i>N$. Choose $N$ so that, also,

$$
\sum_{i=1}^{N}|f(i)|+\left|\sum_{i=1}^{N} \phi(i) f(i)\right|>1+2 \alpha .
$$

There is a $\sigma\left(c_{0}\right)$-neighbourhood $V$ of $f$ such that, for $g \in V$,

$$
\sum_{i=1}^{N}|g(i)|+\left|\sum_{i=1}^{N} \phi(i) g(i)\right|>1+\alpha .
$$


If $g \in V$ and $\langle\phi, g\rangle=0$, then

But

$$
\left|\sum_{N+1}^{\infty} \phi(i) g(i)\right|=\left|\sum_{1}^{N} \phi(i) g(i)\right| .
$$

By (1) and (2),

$$
\left|\sum_{N+1}^{\infty} \phi(i) g(i)\right| \leqq(1+\alpha) \sum_{N+1}^{\infty}|g(i)| \text {. }
$$

$$
\|g\|=\sum_{1}^{N}|g(i)|+\sum_{N+1}^{\infty}|g(i)|>\frac{1+\alpha}{1+\alpha}=1 .
$$

Hence $V$ does not meet $K \cap U^{0}$.

An example is given in (1) (loc. cit.) of a $\sigma\left(c_{0}\right)$-dense linear subspace $E$ of $l_{1}$ (necessarily not a hyperplane) such that $\operatorname{cl}\left(E \cap U^{0}\right)$ contains no multiple of $U^{0}$.

(ii) $X=l_{1}$. We use the following (more or less standard) notation: $e$ denotes the sequence having 1 in each place, and $e_{n}$ denotes the sequence having 1 in place $n$ and 0 elsewhere.

Let $L$ be a linear functional on $m$ such that $L\left(c_{0}\right)=\{0\}, L(e)=1$ and $\|L\|=1$ (i.e. an "extended limit"; the existence of such functionals is guaranteed by the Hahn-Banach theorem). We show that $d\left(L, l_{1}\right)=1$. Choose $x \in l_{1}$. For any $\varepsilon>0$, there exists $N$ such that

$$
\sum_{N+1}^{\infty}|x(n)| \leqq \varepsilon
$$

Let $f_{N}=e-\left(e_{1}+\ldots+e_{N}\right) \in m$. Then $\left\|f_{N}\right\|=1, L\left(f_{N}\right)=1$, and $\left|\left\langle x, f_{N}\right\rangle\right| \leqq \varepsilon$. Hence $\|L-x\| \geqq 1-\varepsilon$.

First let $\phi=L$. Then $K$ contains $c_{0}$, from which it follows that

$$
\operatorname{cl}\left(K \cap U^{0}\right)=U^{0}
$$

(in general, $U$ is $\sigma\left(X^{*}\right)$-dense in $\left.U^{00}\right)$. In this case, $\operatorname{cl}\left(K \cap U^{0}\right)$ is $B(\phi)$.

Now take $k>1$, and let $\phi=L+k e_{1}$. Then $d\left(\phi, l_{1}\right)=1$. We show that:

(a) There exists $f_{0}$ in $\operatorname{cl}\left(K \cap U^{0}\right)$ with $\left\langle\phi, f_{0}\right\rangle=2$.

(b) Given $\varepsilon>0$, there exists $f_{\varepsilon} \notin \mathrm{cl}\left(K \cap U^{0}\right)$ with $\left\langle\left\langle\phi, f_{\varepsilon}\right\rangle\right| \leqq \varepsilon$ and $\left\|f_{\varepsilon}\right\|=1$.

Roughly speaking, this means that $\operatorname{cl}\left(K \cap U^{0}\right)$ goes out as far as $B(\phi)$ in some directions, and only as far as $A(\phi)$ in others.

(a) Let

$$
f_{0}=e-\left(1-\frac{1}{k}\right) e_{1}=\left(\frac{1}{k}, 1,1, \ldots\right)
$$

Then $\left\langle\phi, f_{0}\right\rangle=2$, and $f_{0}$ is the $\sigma\left(l_{1}\right)$-limit of the sequence $\left(f_{n}\right)$, where

$$
f_{n}=\left(\frac{1}{k}, 1, \ldots, 1,-1,-1, \ldots\right)=-e+\left(1+\frac{1}{k}\right) e_{1}+\sum_{j=2}^{n} 2 e_{j} \text {. }
$$


WEAK-STAR CLOSURE OF UNIT BALL IN A HYPERPLANE 11 Each $f_{n}$ is in $K \cap U^{0}$.

(b) We may assume that $\varepsilon \leqq k-1$. Let

$$
f_{\varepsilon}=\left(\frac{1+\varepsilon}{k},-1,-1, \ldots\right)=\left[1+\frac{1}{k}(1+\varepsilon)\right] e_{1}-e .
$$

Then $\left\langle\phi, f_{\varepsilon}\right\rangle=\varepsilon$. If $\|g\| \leqq 1$ and $g(1)>\frac{1}{k}\left(1+\frac{\varepsilon}{2}\right)$, then $\left\langle k e_{1}, g\right\rangle>1+\frac{\varepsilon}{2}$, while $|\langle L, g\rangle| \leqq 1$, so $\langle\phi, g\rangle \neq 0$. Hence $f_{\varepsilon} \notin \mathrm{cl}\left(K \cap U^{0}\right)$.

\section{REFERENCES}

(1) N. BourbaKI, Espaces Vectoriels Topologiques, chap. III, IV, V (Paris, 1955).

(2) M. M. DAY, Normed Linear Spaces (Berlin, 1962).

(3) G. J. O. JAMESON, The duality of pairs of wedges, Proc. London Math. Soc. (3) 24 (1972), 531-547.

(4) D. R. KERR, Seminorm-dual subspaces of the algebraic dual of a linear space, Math. Z. 104 (1968), 222-225.

UNIVERSITY OF WARWICK

COVENTRY 